\title{
VHL Expression Level in the Pathological Tissue is Significantly Associated with Clinical Outcomes of Platinum-Based Chemotherapy in Non-Small Cell Lung Cancer Patients.
}

\section{Yejun Cao}

Tongji University

Lingwei Wang

Tongji University

Qiying Zhang

Tongji University

Qiyan Zhang

Shangrao Normal University

Yang Han

Tongji University

Jie Liu

Tongji University

Jinyi Wang

Tongji University

Liang Ma

Tongji University

Zhengliang Sun

Tongji University

Tian Zhao

Tongji University

Guangxue Wang

Shanghai East Hospital

Qinchuan Li

Shanghai East Hospital

Guohan Chen

Tongji University

Xuan Hong ( $\nabla$ hxseh@126.com )

Tongji University 
Research

Keywords: VHL, NSCLC, platinum-based chemotherapy, clinical outcome

Posted Date: March 19th, 2021

DOI: https://doi.org/10.21203/rs.3.rs-337079/v1

License: (c) (i) This work is licensed under a Creative Commons Attribution 4.0 International License. Read Full License 


\section{Abstract}

Objective: To investigate the association between expression level of Von Hippel-Lindau(VHL) in pathological tissue and outcomes of platinum-based chemotherapy in non-small cell lung cancer (NSCLC) patients.

Methods: The pathological samples of NSCLC patients were obtained for immunohistochemical staining to evaluate the expression level of VHL. Furthermore, their clinical data were collected and prognosis was traced by phone. The correlation between gene expression level and the hematotoxicity was evaluated by chi-square test. The influence of VHL expression level on the risk of hematotoxicity was tested by logistic regression model. The survival curve was plotted by Kaplan-Meier method and the survival rate between the two groups was compared by log-rank test.

Results: A total of 110 NSCLC patients were enrolled in this study, the median follow-up time of these patients was 27.5 months. In the whole group, 31 patients had died by the last date of follow-up to get their survival information (Nov.10,2020), with a median survival time of 24.3 months. Though immunohistochemical analysis,we found that 59 patients(53.6\%) had weak expression level of VHL or lack of expression in their tumor tissues, while 51 patients(46.4\%) presented moderate or high expression. We found that the patients with weak expression of VHL in their carcinoma tissue or lack of expression had more opportunities to occur neutropenia after platinum-based chemotherapy $(\mathrm{OR}=0.264,95 \% \mathrm{Cl}=0.085-0.818, P$-value $=0.021)$. And the expression level of $\mathrm{VHL}$ was correlated with OS(Logrank test $\triangle P$-value $=0.007 \otimes \mathrm{HR}=4.219,95 \% \mathrm{Cl}$ : 1.75-10.174, $P$-value $=0.001)$, while not

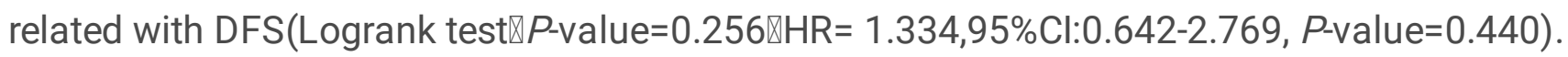

Conclusion: The expression level of VHL gene in pathological tissue is related with Granulocytosis and leukocytotoxicity after platinum-based chemotherapy in NSCLC patients. It can be used as a biomarker to predict the risk of neutropenia and the prognosis of NSCLC patients.

\section{Introduction}

Lung cancer is still a leading cause of the morbidity and mortality generated by cancers all over the world. The main pathological type of lung cancer is non-small cell lung cancer (NSCLC) which accounts for about $85 \%$ of lung cancer. ${ }^{[1,2]}$ It is often diagnosis too late to accept surgery for a large proportion of NSCLC parents. Instead, they have to accept chemotherapy to prolong their survival time. Fortunately, the discovery of immunological therapy such as programmed death molecule-1 (PD-1) and its ligand (PD-L1) has significantly improved survival times of NSCLC patients. However, the promotion of immunological therapy is restricted by its low objective response rate and its expensive expense in China. Furthermore, platinum-based chemotherapy is often used in combination with immunotherapy, and platinum-based chemotherapy is also the first-line treatment for lung cancer. Therefore, the investigation on the platinumbased chemotherapy in cancer treatment still need to be performed. ${ }^{[1,4,5]}$ 
Chemotherapeutic drugs for NSCLC, represented by platinum-based drugs, mainly kill tumor cells

extensively through the way of DNA damage, which inevitably cause damage to normal cells. ${ }^{[4,5,12]}$ The most common side effects in clinical are hematotoxicity and gastrointestinal reactions. The blood toxicity, including neutropenia and leukopeniais, is the most common toxicity in platinum chemotherapy. It not only affects the quality of NSCLC patients' lives, but also has a great impact on the follow-up treatment.

VHL is a classic tumor suppressor gene, located on the short arm of chromosome 3(3p25-26). ${ }^{[7]}$ Many researches have pointed out that $\mathrm{VHL}$ could influence the occurrence and metastasis of carcinomas through regulating the HIF pathway. ${ }^{[6-9]}$ Several studies showed that lack of VHL could cause resistance in the chemotherapy of different carcinomas, including clear-cell renal cell carcinoma (ccRCC) and other solid tumors. ${ }^{[7,10]}$ However, little is known about the correlation between NSCLC chemotherapy and VHL gene. ${ }^{[7-10]}$

The aim of the current study is to investigate the association between Von Hippel-Lindau(VHL) expression and outcomes of platinum-based chemotherapy in NSCLC patient. Specially, we evaluated the expression of VHL in the pathological tissue of NSCLC patients by IHC analysis. Given the fact that the biomarkers, to predict the risk of blood toxicity after platinum-based chemotherapy, are scarce for clinical use, our ongoing work on VHL has significant implications in the treatment of NSCLC.

\section{Materials And Methods}

\section{Ethical approval}

The procedures involved in our study conformed to the ethical standards of research Committee in Shanghai East Hospital. It is consistent with the ethical standards of the 1964 Helsinki Declaration and its subsequent revisions. All patients in the study were notified and given written consent. No animals were used in our research. All experiments involved in our study were conducted in accordance with approved guidelines and regulations.

\section{Study cohorts}

In this study, 110 patients who accepted first-line platinum-based double chemotherapy plan were recruited from January 2016 to July 2020. All patients received radical surgery and were diagnosed as NSCLC by pathology when enrolled. The pathological tissue samples in our study were taken from NSCLC patients who were underwent radical operation in Shanghai East Hospital from 2016 to 2020.Then,we evaluated the association with the expression of $\mathrm{VHL}$ gene in histopathological specimen and clinical outcomes in NSCLC patients treated by platinum-based chemotherapy.

\section{Treatment}


110 NSCLC patients in our study were treated with platinum-based chemotherapy,including double-DNA damage agents(cisplatin or nedaplatin combined with gemcitabine or pemetrexed) and platinum-tubulin targeting agents(cisplatin or nedaplatin combined with docetaxel).They received above plan for two to four cycles. The usages for cisplatin and nedaplatin were $75 \mathrm{mg} / \mathrm{m} 2$ on day 2 every three weeks and $100 \mathrm{mg} / \mathrm{m} 2$ on day 2 every three weeks. The usages for gemcitabine and pemetrexed were $1000 \mathrm{mg} / \mathrm{m} 2$ on day 1 and day 8 every three weeks and $500 \mathrm{mg} / \mathrm{m} 2$ on day 1 every three weeks. $75 \mathrm{mg} / \mathrm{m} 2$ on day 2 every three weeks was for docetaxel.

\section{Specimen collection and immunohistochemistry assay}

The tissue samples in our study were taken from NSCLC patients who were underwent radical operation in Shanghai East Hospital from 2016 to 2020. Immunohistochemical streptavidin-perosidase『SP『method was used to detect pVHL in tumor tissue(Figure 1). The paraffin sections were dewaxed and dehydrated regularly, repaired under high pressure, sealed with hydrogen peroxide, incubated with primary antibody in a refrigerator at $4^{\circ} \mathrm{C}$, and incubated with secondary antibody. Then,DAB reagent was used for color development, restained with hematoxylin, differentiated with hydrochloric acid alcohol, washed to return to blue, and sealed with neutral gum. The result was evaluated by pathologists of Shanghai East Hospital through semi-quantitative counting method in a blinding method.

\section{Toxicity}

Chemotherapy toxicities were evaluated third weekly based on the National Cancer Institute ( $\mathrm{NCl})$. No toxicities and Grades 1 were defined to negative or weak toxic effects.Grades 2, 3 and 4 was defined to severe ones. Grade 5, never observed in our study, meant death.

\section{Statistical analysis}

We used the SPSS version 26.0 software to analyse the data. Chi-squrared test was used for illustrating the relevance between the expression level of VHL and blood toxicity of platinum-based chemotherapy. Furthermore,we used Logistic regression to evaluate its trend. The Kaplan-Meier curves and Cox regression analysis were used for illustrating the association between the expression level of VHL and patients' OS/PFS. Statistical results were taken $=0.05$ as the test level, and $\mathrm{P}<0.05$ was considered statistically significant.

\section{Results}

\section{Clinical characteristics of patients}

The subjects, including 110 NSCLC patients, in our study were recruited from Shanghai East Hospital between January 2016 to July 2020 . The clinical characteristics of them are summarized in Table1.Though immunohistochemical analysis,we found that 59 patients(53.6\%) had weak expression level of $\mathrm{VHL}$ or lack of expression in their tumor tissues, while 51 patients $(46.4 \%)$ presented moderate or high expression. We compare the relationship between the expression level of $\mathrm{VHL}$ and patients' clinical 
characteristics, including age, gender, smoking status, histology of carcinoma, clinical stage for lung cancer and the size of carcinoma. The result showed that Age could influence the expression level of $\operatorname{VHL}(P$-value $=0.024)$, while other clinical features are not correlated with the expression of $\mathrm{VHL}(P$ value $>0.05)$.

\section{The expression level of VHL was correlated with neutropenia}

The association among clinical characteristics of NSCLC patients, the expression level of VHL in carcinoma's tissue and the incidence of granulocytopenia occurred in NSCLC patients who received platinum-based chemotherapy was calculated by Chi-squrared test. We found that the expression of VHL was related to the neuttropenia after platinum-based therapy $(P$-value $=0.005)$, and other clinical features were not associated with neutropenia( $P$-value $>0.05)$. Then, we further research above factors through multivariate regression analysis. The result showed that the patients with negative or weak expression of VHL had more opportunities to occur neutropenia after platinum-based chemotherapy $(\mathrm{OR}=0.264,95 \% \mathrm{Cl}=0.085-0.818, P$-value $=0.021)$. More details are outlined in Table 2 .

On the other way, the calculation through Chi-squrared test showed that the expression of $\operatorname{VHL}\left(X^{2}=5.044, P\right.$-value $\left.=0.025\right)$ and gender $\left(X^{2}=4.632, P\right.$-value $\left.=0.031\right)$ were related with leukopenia caused by platinum-based chemotherapy. Further study through multivariate regression analysis showed that the VHL expression level $(\mathrm{OR}=0.315,95 \% \mathrm{Cl}=0.088-1.132, P$-value $=0.077)$ and gender $(\mathrm{OR}=4.088,95 \% \mathrm{Cl}=1.012-$ $16.52, P$-value $=0.048$ ) were not the determining element for the leukopenia.

\section{Association with progression-free survival (PFS) or overall survival (OS)}

We utilized the Kaplan-Meier curves to analyze the relationship between the expression level of VHL and DFS or OS(Figure 2). In the whole group, 87 subjects with 8 ones lost connection were observed,31 patients had died by the last date of follow-up to get their survival information (Nov.10,2020), with a median survival time of 24.3 months.

We found that the expression of VHL was correlated with OS(Logrank test $\triangle \mathrm{P}$-value $=0.007$ ), while not related with DFS(Logrank test $\triangle \mathrm{P}$-value $=0.256)$. Furthermore, We researched the association between VHL expression level and OS. The result of COX regression analysis showed that persons with high expression of VHL in their pathological tissue had a worse prognosis than those with negative or weak expression $(\mathrm{HR}=4.219,95 \% \mathrm{Cl}: 1.75-10.174, \mathrm{P}$-value $=0.001)$. We also found that the higher the TMN stage, the worse the prognosis $(\mathrm{HR}=4.96,95 \% \mathrm{Cl}: 1.902-12.932$, $\mathrm{P}$-value=0.001). The result was summarized in Table 3 .

Our research also found that TMN stage could influence DFS(HR=5.072,95\%Cl: $2.101-12.244, \mathrm{P}-$ value $<0.001)$,however,the expression level of VHL was not related with PFS(HR=1.334,95\%Cl: $0.642-$ 2.769, P-value=0.440).

\section{Discussion}


In this study,we found that the expression level of VHL in the tumor tissue of NSCLC patients plays an important role in predicting the risk of Granulocytosis in platinum-based chemotherapy. According to IHC staining result, we confirmed that granulocytosis is more likely to happen in the person whose pathological tissue is lack or weak expression of VHL. Previous result has suggested that VHL is a classic tumor suppressor gene, which plays a significant role in the occurrence and progression of many cancers including clear-cell renal cell carcinoma(ccRCC),colon cancer,pancreatic cancer and so on. Early research showed that the absence of VHL led to the continuous accumulation of HIF-1a, which in turn leads to the continuous opening of HIF pathway. ${ }^{[6-8]}$ This results in continuous overexpression of downstream factors such as vascular endothelial growth factor (VEGF), which causes to high vascularization in normal tissues and promotes the production of erythropoietin (EPO), ultimately evolking the growth and proliferation of carcinoma and hindering the degradation of tumor cells. Furthermore, recent studies have shown that the HIF pathway mediated by VHL is closely associated with the resistance of multiple chemotherapeutic drugs and radiotherapy. ${ }^{[7,16]} \mathrm{VHL}$ regulates the HIF pathway, and leads to the overexpression of MDR (multi-drug resistance) gene, which has been widely proved to be related with the resistance of cisplatin, vinblastine, anthracycline, taxane and other chemotherapy drugs treated with some hypoxic carcinomas. However, the correlation between VHL gene and NSCLC chemoradiotherapy and prognosis has rarely been reported. ${ }^{[10-13,18]}$

For this reason, we evaluated the relationship between clinical characteristics of NSCLC patients and the expression level of VHL in their carcinoma tissues. Then, we explored the association among the protein expression level, granulocytosis and OS/DFS. In current study, we found that VHL was more likely to be absent or poorly expressed in NSCLC tissues and Age could influence the expression level of VHL( $P$ value $=0.024)$. This finding may further support the description of VHL low expression or lack in tumor cells in previous studies. We also discovered that the expression of VHL was related to the neutropenia after platinum-based therapy $(P$-value $=0.005)$, further multivariate regression analysis showed that lack or low expression of $\mathrm{VHL}$ increased the risk of neutropenia after platinum-based chemotherapy. At present, the mechanism of VHL expression level affecting hemotoxicity is still under study. We speculate that the reason for this phenomenon may be related to the regulation of VHL-mediated HIF pathway and its downstream genes on apoptosis in vivo, which is closely similar to the mechanism of MMP2 gene mediating NSCLC granulocyte toxicity. ${ }^{[21,22]}$ In addition, we found that the expression of $\operatorname{VHL}\left(X^{2}=5.044, P\right.$ value $=0.025)$ and gender $\left(X^{2}=4.632, P\right.$-value $\left.=0.031\right)$ may be related with leukopenia caused by platinumbased chemotherapy. However, multivariate regression analysis didn't support this in an individual prognostic factor, we planned to increase the sample cohort to verify it.

Furthermore, through the Kaplan-Meier curves, we explored the relationship between the expression level of VHL and DFS or OS. The conclusion showed that VHL expression level(Logrank test $\triangle P$-value= $0.007, \mathrm{HR}=4.219,95 \% \mathrm{Cl}: 1.75-10.174, P$-value $=0.001)$ was correlated with OS (Logrank test $\triangle P$-value $=$ 0.007 , while it did not related with DFS(Logrank test $\triangle P$-value $=0.256, \mathrm{HR}=1.334,95 \% \mathrm{Cl}: 0.642-2.769, P$ value $=0.440$ ). The person who had negative or weak expression in his tumor tissue could live longer than the one who had moderate or high expression in the tissue.Therefore, the expression of VHL in the 
pathological tissue may be a useful tool for early prediction of the hematotoxicity of platinum-based chemotherapy and the outcome of NSCLC patients. Unlike our study, a previous trial showed that loss of VHL might cause the poor survival of lung adenocarcinoma. However, unlike the previous trial, our study

had a considerably larger population and more various patients with different pathological pattern. ${ }^{23]}$ The difference in patient group may account for the different results. Because the expression level of VHL was effective in predicting the risk of neutropenia in platinum-based chemotherapy, it clearly that the polymorphisms of VHL in blood has potential as a prediction site for the hemotoxicity of platinum-based chemotherapy. Further studies need to be done, however, to firmly establish its place as a predictive marker for hematotoxicity of platinum-based chemotherapy in NSCLC patients.

In summary, severe neutropenia caused by platinum-based chemotherapy often results in severe infection, greatly destroying the patient's quality of life and effecting the subsequent treatment. In this study, we found that the expression level of VHL gene intumor tissues could be used as a biomarker to predict the risk of neutropenia. If clinical transformation could be achieved, it would be of great significance for individual therapy with platinum drugs. At the same time, VHL expression level could indicate the prognosis of NSCLC patients receiving chemotherapy, which can be used to evaluate the long-term prognosis of patients receiving chemotherapy.

\section{Abbreviations}

VHL: Von Hippel-Lindau; NSCLC: non-small cell lung cancer; PD-1: programmed death molecule-1; ccRCC: clear-cell renal cell carcinoma; SP: streptavidin-perosidase; NCl: National Cancer Institute; DFS囚disease

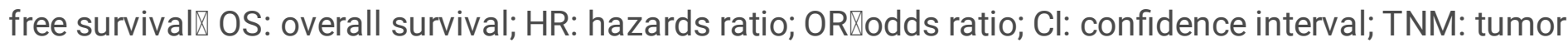
node metastasis; Acknowledgements; VEGF: vascular endothelial growth factor; EPO :the production of erythropoietin; MMP2: matrix metallopeptidase 2 ; MDR: multi-drug resistance

\section{Declarations}

\section{Acknowledgements}

We sincerely thank Pathological Laboratory of Shanghai East hospital for

providing the help in immunohistochemistry and results interpretation.

\section{Authors' contributions}

Yejun Cao, Qiying Zhang,Liang Ma, Jie Liu,Jinyi Wang,Zhengliang Sun,Qinchuan Li and Tian Zhao prepared the sample,Yejun Cao,Lingwei Wang and Qiyan Zhang carryed out the data analysis.Yang Han evaluated the result of immunohistochemistry.Yejun Cao, Lingwei Wang and Xuan Hong collected and checked the data of patients.Yejun Cao, Guohan Chen and Xuan Hong designed the project and wrote the manuscript. 


\section{Ethics approval and consent to participate}

The study was performed in accordance with the Declaration of Helsinki and was approved by the Ethics Committee of Shanghai East hospital.

\section{Funding}

This work is supported by Health Special Project of Health Bureau of Shanghai(Grant No. 201740220) to X Hong; Health Special Project of Pudong Health Bureau of Shanghai(Grant No. PKJ2017-Y21) to Y Han ;Health Special Project of Pudong Health Bureau of Shanghai(Grant No. PW2020E-5) to QC Li ; the National Natural Science Foundation of China (Grant No. 81773266) to QC Li.

\section{Availability of data and materials}

There is no supporting data available.

\section{Consent for publication}

A written informed consent was obtained from the patient for publication of

her clinical details and clinical images in this article.

\section{Competing interests}

The authors declare that they have no competing interests.

\section{References}

1. Chen J, Yin J, Li X, et al. WISP1 polymorphisms contribute to platinum-based chemotherapy toxicity in lung cancer patients. Int J Mol Sci. 2014;15:21011-27.

2. Chen W. Cancer statistics: updated cancer burden in China. Chin J Cancer Res. 2015;27:1.

3. Chen W, Hill H, Christie A, et al. Targeting renal cell carcinoma with a HIF-2 antagonist. Nature. 2016;539:112-7.

4. Han Y, Liu J, Sun M, et al. A Significant Statistical Advancement on the Predictive Values of ERCC1 Polymorphisms for Clinical Outcomes of Platinum-Based Chemotherapy in Non-Small Cell Lung Cancer: An Updated Meta-Analysis. Dis Markers. 2016;2016:7643981.

5. Johnstone TC, Suntharalingam K, Lippard SJ. The Next Generation of Platinum Drugs: Targeted Pt(II) Agents, Nanoparticle Delivery, and Pt(IV) Prodrugs. Chem Rev. 2016;116:3436-86.

6. Du W, Zhang L, Brett-Morris A, et al. HIF drives lipid deposition and cancer in ccRCC via repression of fatty acid metabolism. Nat Commun. 2017;8:1769.

7. Gossage L, Eisen T, Maher ER. VHL, the story of a tumour suppressor gene. Nat Rev Cancer. 2015;15:55-64. 
8. Kang MR, Park KH, Lee CW, et al. Small activating RNA induced expression of VHL gene in renal cell carcinoma. Int J Biochem Cell Biol. 2018;97:36-42.

9. Lenglet $\mathrm{M}$, Robriquet $\mathrm{F}$, Schwarz $\mathrm{K}$, et al. Identification of a new VHL exon and complex splicing alterations in familial erythrocytosis or von Hippel-Lindau disease. Blood. 2018;132:469-83.

10. Li S, Rodriguez J, Li W, et al. EgIN3 hydroxylase stabilizes BIM-EL linking VHL type $2 \mathrm{C}$ mutations to pheochromocytoma pathogenesis and chemotherapy resistance. Proc Natl Acad Sci U S A. 2019;116:16997-7006.

11. Lv C, Bai Z, Liu Z, et al. Renal cell carcinoma risk is associated with the interactions of APOE, VHL and MTHFR gene polymorphisms. Int J Clin Exp Pathol 2015.

12. Qian $C Y$, Zheng $Y$, Wang $Y$, et al. Associations of genetic polymorphisms of the transporters organic cation transporter 2 (OCT2), multidrug and toxin extrusion 1 (MATE1), and ATP-binding cassette subfamily $\mathrm{C}$ member 2 ( $\mathrm{ABCC} 2$ ) with platinum-based chemotherapy response and toxicity in nonsmall cell lung cancer patients. Chin J Cancer. 2016;35:85.

13. Tarade D, Ohh M. The HIF and other quandaries in VHL disease. Oncogene. 2018;37:139-47.

14. Wang WC, Tsou MH, Chen HJ,et al. Two single nucleotide polymorphisms in the von Hippel-Lindau tumor suppressor gene in Taiwanese with renal cell carcinoma. BMC Res Notes. 2014;7:638.

15. Chen G, Zhou J, Chen J, et al. VHL regulates NEK1 via both HIF-2alpha pathway and ubiquitinproteasome pathway in renal cancer cell. Biochem Biophys Res Commun. 2019;509:797-802.

16. Chen W, Hill H, Christie A, et al. Targeting renal cell carcinoma with a HIF-2 antagonist. Nature. 2016;539:112-7.

17. Ding XF, Zhou J, Chen G, Wu YL. VHL loss predicts response to Aurora kinase A inhibitor in renal cell carcinoma cells. Mol Med Rep. 2018;18:1206-10.

18. Wu K, House L, Liu W,et al. ersonalized targeted therapy for lung cancer. Zhongguo Fei Ai Za Zhi. 2013;16:C21-34.

19. Wu K, House L, Liu W,et al. Personalized targeted therapy for lung cancer. Chin J Lung Cancer. 2013;16:C21-34.

20. Yin JY, Li X, Li XP, et al. Prediction models for platinum-based chemotherapy response and toxicity in advanced NSCLC patients. Cancer Lett. 2016;377:65-73.

21. Zhao X, Wang X, Wu W, et al. Matrix metalloproteinase-2 polymorphisms and clinical outcome of Chinese patients with nonsmall cell lung cancer treated with first-line, platinum-based chemotherapy. Cancer. 2012;118:3587-98.

22. Wu DM, Deng SH, Liu T,et al. TGF- $\beta$-mediated exosomal Inc-MMP2-2 regulates migration and invasion of lung cancer cells to the vasculature by promoting MMP2 expression. Cancer Med. 2018 Oct;7(10):5118-29.

23. Zhao YS, Le Y, Wang SF,et al. The relationship between EGFR gain and VHL loss in lung adenocarcinoma and poor patient survival. Int J Clin Oncol. 2011 Dec;16(6):679-85. 


\section{Tables}

Due to technical limitations, table 1, 2 and 3 are only available as a download in the Supplemental Files section.

\section{Figures}
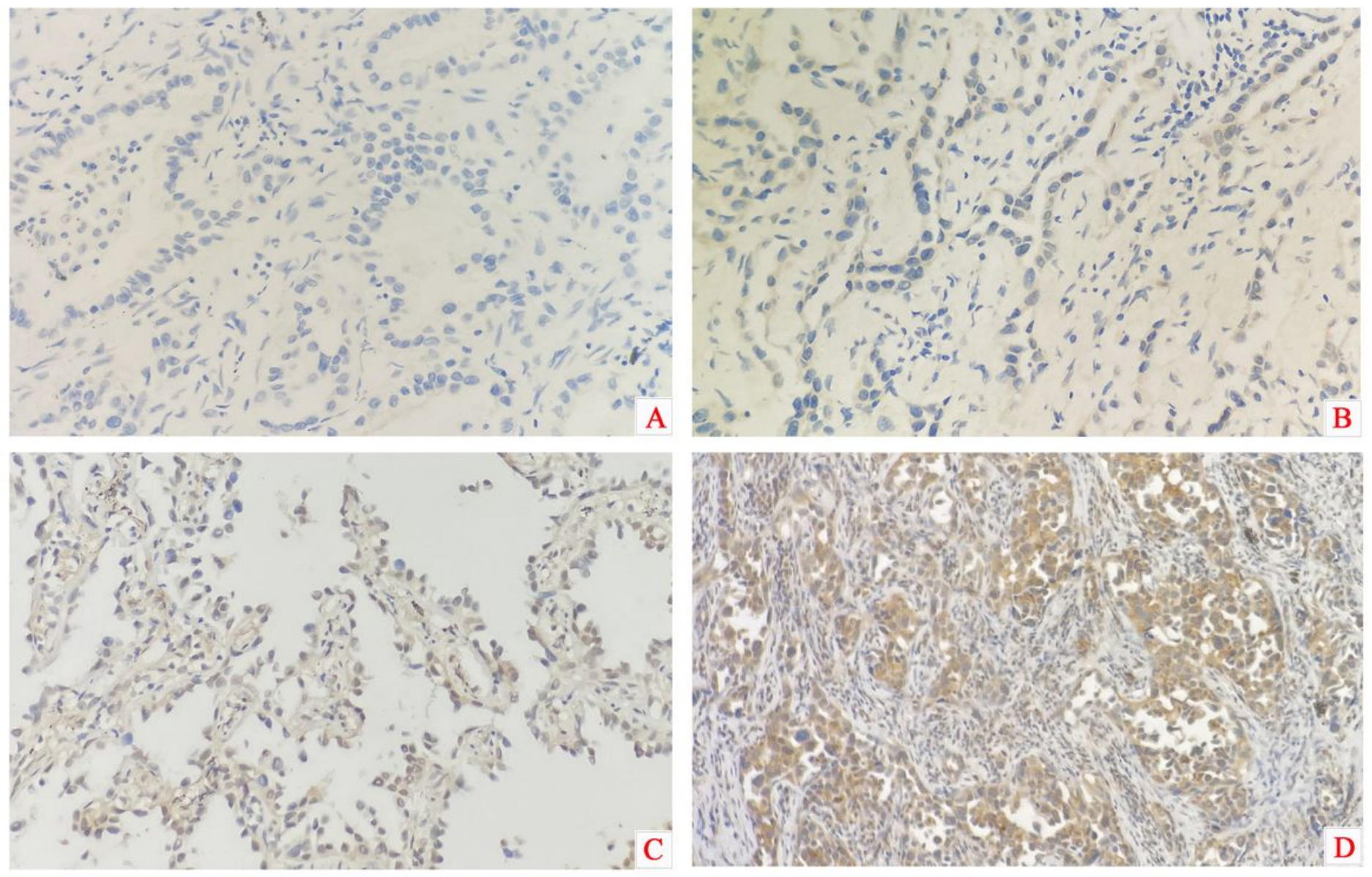

\section{Figure 1}

Representative IHC staining for VHL expression in non-small cell lung cancer (NSCLC). A, negative expression of VHLin tumor tissue. B, Weak expression of VHL. C『Moderate expression of VHL D, High expression of VHL., $\times 200$ 

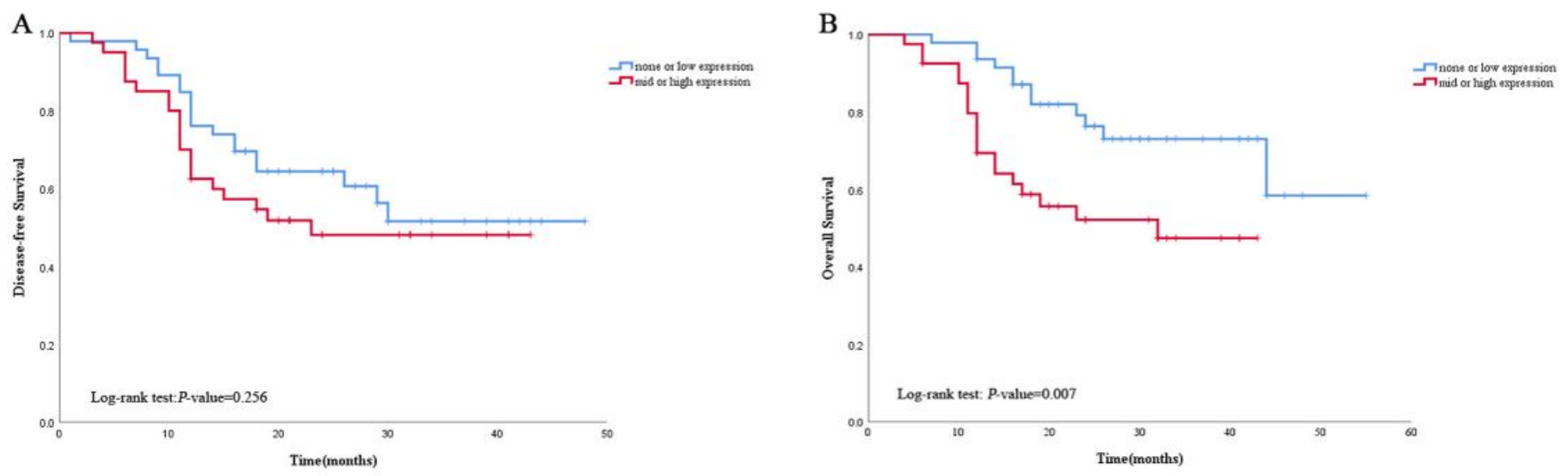

Figure 2

Kaplan-Meier survival curves for patients with non-small cell lung cancer (NSCLC) according to VHL expression level. A, Disease-free Survival (DFS) of patients with non-small cell lung cancer (NSCLC) receiving platinum-based chemotherapy. B, Overall survival(OS) of patients with non-small cell lung cancer (NSCLC) receiving platinum-based chemotherapy.

\section{Supplementary Files}

This is a list of supplementary files associated with this preprint. Click to download.

- Table1.pdf

- Table2.pdf

- Table3.pdf 